\title{
Antimicrobial resistant Helicobacter fennelliae isolated from non-diarrheal child stool sample in Battambang, Cambodia
}

\author{
Supaporn Ruksasiri ${ }^{1}$, Woradee Lurchachaiwong ${ }^{1 *}$, Patcharawalai Wassanarungroj ${ }^{1}$, Oralak Serichantalergs ${ }^{1}$, \\ Chiek Sivhour ${ }^{2}$, Nou Samon ${ }^{3}$, Sovann Ly ${ }^{4}$ Lon Chanthap ${ }^{3}$, Ladaporn Bodhidatta ${ }^{1}$ and John Crawford ${ }^{1}$
}

\begin{abstract}
Helicobacter fennelliae (H. fennelliae) is associated with human gastroenteritis; however, H. fennelliae was isolated and confirmed by phenotypic and genotypic identification from a non-diarrheal child stool sample in Cambodia. Antimicrobial susceptibility testing demonstrated that this isolate had a high minimal inhibitory concentration against macrolides and quinolones, which are first-line antibiotic treatment choices for Campylobacter infections. Consequently, macrolides and quinolones were likewise expected to be ineffective against Campylobacter-like organisms such as $\mathrm{H}$. fennelliae. This isolate warranted further genetic characterization to better understand associated antibiotic resistance mechanisms. Resistant pathogens from asymptomatic diarrheal cases are likely underestimated, and as such colonized individuals may spread resistant organisms to local community members and the environment.
\end{abstract}

Keywords: Helicobacter fennelliae, Non-diarrheal sample, Child, Cambodia, First-report

\section{Background}

Helicobacter fennelliae (H. fennelliae) is a new Campylobacter species originally isolated from asymptomatic, homosexual men with enteritis and proctitis in the past few decades [1]. Like $H$. cinaedi, this species is classified as enterohepatic Helicobacter that inhabits and causes bacteremia in intestinal and hepatobiliary tracts of various mammal and other species [2]. Additional evidence suggests that $H$. fennelliae was implicated as a contributing cause of human proctocolitis, gastroenteritis, and bacteremia, particularly in immunocompromised individuals [2,3]. This Helicobacter species is a fastidious organism that is likely underestimated, and little is known about routes of transmission other than evidence indicates it is a zoonotic infection [2]. As a fastidious organism, molecular genotyping methods are recommended to identify Helicobacter species. Towards that end, the groEL and hsp60 genes encode a $60 \mathrm{kDa}$

\footnotetext{
*Correspondence: woradeel.fsn@afrims.org

${ }^{1}$ Department of Enteric Diseases, Armed Forces Research Institute

of Medical Sciences, 315/6 Rajvithi Road, Bangkok 10400, Thailand

Full list of author information is available at the end of the article
}

chaperonin protein present in virtually all eubacteria, some archaea, and in the plastids and mitochondria of eukaryotes. The utility of this target for bacterial species identification, detection, quantification, phylogenetic analysis, and microbial community profiling was well established [4]. Treatment recommendation guidelines are still not available for enterohepatic Helicobacter species. Various individual and combined antibiotic regimens were successfully used in treating Helicobacter infections; however, there is insufficient information to determine resistance rates of $H$. fennelliae. The main objective of this report is to describe phenotypic, genotypic, and antimicrobial susceptibility (AST) data from this $H$. fennelliae isolate from the stool of non-diarrheal child in Cambodia.

\section{Methods}

A surveillance study to describe diarrhea etiologic agents in children and military personnel in Battambang, Cambodia has been conducted from 2014 until present. Both diarrheal and non-diarrheal stool samples were observed by microscopic examination for the presence of parasites, protozoa, and larvae. Samples were also assessed for the 
presence of Giardia, Cryptosporidium by enzyme-linked immunosorbent assay (ELISA), and for diarrheagenic $E$. coli by polymerase chain reaction (PCR) [5]. Enteric pathogens, including Campylobacter species, were isolated and identified by traditional culture methods [6]. The suspected Campylobacter-like colonies were subcultured on blood agar supplemented with $6 \%$ sodium formate and fumarate for $48-72 \mathrm{~h}$ at $37^{\circ} \mathrm{C}$ under microaerobic conditions $\left(10 \% \mathrm{CO}_{2}\right.$ and $\left.5 \% \mathrm{O}_{2}\right)$. The biochemical identifications were included oxidase, catalase, indoxyl hydrolysis, hippurate hydrolysis, nitrate reduction, urease, hydrogen sulfide production, susceptibility to cephalothin and nalidixic acid (30 $\mu \mathrm{g}$ disc) (BD, Spark, USA), oxygen and temperature tolerance test. According to no antimicrobial susceptibility recommendation guidelines, $H$. fennelliae resistance was determined using the minimal inhibitory concentration (MIC) by E test (Liofilchem, Roseto degli Abruzzi TE, Italy) against azithromycin (AZM), erythromycin (ERY), nalidixic acid (NAL), ciprofloxacin (CIP), levofloxacin (LEV), ceftriaxone (CRO), spectinomycin (SPT), and tetracycline (TET). C. jejuni ATCC 33560 was used as a quality control strain.

Genomic DNA of suspected Campylobacter-like colonies was extracted and subsequently confirmed as belonging to the Campylobacter genus by screening for the 16S rRNA gene [7]. To determine Campylobacter species, the 15 primer sets of cpn 60 target gene were used for verified species as described elsewhere [7, 8]. Subsequently, the unknown Campylobacter species beyond 15 primer sets identification were further sequencing analysis by amplifying cpn 60 target gene with degenerate primers $\mathrm{H} 729$ and H730 [4]. The sequences of degenerate primers were H729: 5'-CGCCAGGGTTTTCCCAGT CACGACGAIIIIGCIGGIGAYGGIACIACIAC- $3^{\prime}$ and H730 5'-AGCGGATAACAATTTCACACAGGAYKIYKITCICCRAAI CCIGGIGCYTT-3'. PCR amplification was carried out in a total volume of $50 \mu \mathrm{L}$ containing $6 \mu \mathrm{L}$ of genomic DNA template, $2.5 \mathrm{U}$ AmpliTaq Gold ${ }^{\circledR}$ DNA polymerase (Applied Biosystems, Foster City, Calif.), $5 \mathrm{mM} \mathrm{MgCl}_{2}, 100 \mu \mathrm{M}$ each of the dNTPs and $50 \mathrm{nM}$ each of degenerate primers [4]. The cycling conditions were performed at $94{ }^{\circ} \mathrm{C}$ for $5 \mathrm{~min}$, followed by 28 cycles of
1 min at $94{ }^{\circ} \mathrm{C}, 1 \mathrm{~min}$ at $46^{\circ} \mathrm{C}, 1 \mathrm{~min}$ at $72{ }^{\circ} \mathrm{C}$, and a final extension at $72{ }^{\circ} \mathrm{C}$ for $10 \mathrm{~min}$. The purified PCR products were additionally differentiate Campylobacter species from Helicobacter and Acrobacter species using primers M13F-pUC (-40) 5'-GTTTTCCCAGTCACGAC-3' and M13R (-20) 5'-GCGGA-TAACAATTTCACACAGG-3'. The result of partial cpn 60 sequences $(555 \mathrm{bp})$ was compared with the database in cpnDB (http://cpndb.cbr.nrc. ca) [4]. The confirmed partial sequence was submitted to the National Center for Biotechnology Information (NCBI) before constructing phylogenetic analysis by BioNumerics software version 7.6 (Applied Maths, Belgium).

\section{Results and discussion}

A non-diarrheal stool sample of a young child who presented to the hospital with fever and, cough was submitted for laboratory testing. The stool characteristic was loose without mucus, blood, RBCs, or WBCs. No gastrointestinal parasites were detected microscopically or by ELISA. Other enteric bacterial pathogens, including diarrheagenic $E$. coli, were not identified, except for suspected colonies of a Campylobacter-like organism. The colonies characteristics which were presented after 6 days incubation were thin, flat, film-like colony, with a hypochlorite odor. Biochemical reactions of the colony were positive for oxidase, catalase, and indoxyl acetate hydrolysis. It was susceptible to cephalothin disk but resistant to nalidixic acid disk and could be grown at $42{ }^{\circ} \mathrm{C}$ under microaerobic conditions. Culture results indicated that $H$. fennelliae grows well by supplementing $6 \%$ sodium formate and fumarate in blood agar. This is likely due to the fact that formate replaces hydrogen as the electron donor, and fumarate serves as the terminal electron acceptor for hydrogen-required organism growth [9]. Notably, an absence of hydrogen, the low-cost supplemented media, and a long incubation period are suggested to support growth of $H$. fennelliae.

The MIC results of $H$. fennelliae and C. jejuni ATCC 33560 were presented in Table 1 . Results for this $H$. fennelliae isolate demonstrated high MICs to macrolides and quinolones, consistent with previous studies $[10,11]$ and similar to $H$. cinaedi data [12]. Macrolides, generally

Table 1 Determination the minimal inhibitory concentration (MIC) results of $H$. fennelliae and C. jejuni ATCC 33560 against azithromycin (AZM), erythromycin (ERY), nalidixic acid (NAL), ciprofloxacin (CIP), levofloxacin (LEV), tetracyclin (TET), ceftriaxone (CRO) and spectinomycin (SPT)

\begin{tabular}{llllllll}
\hline Isolates & \multicolumn{1}{l}{ MIC $(\boldsymbol{\mu} \mathbf{g} / \mathbf{m L})$} & & & & \\
\cline { 2 - 8 } & AZM & ERY & NAL & CIP & LEV & TET & CRO \\
\hline H. fennelliae & $\geq 256$ & $\geq 256$ & $\geq 256$ & $\geq 32$ & 3 & 0.125 & 0.125 \\
C. jejuni ATCC33560 & 0.125 & 0.75 & 4 & 0.094 & 0.25 & 0.25 & $\geq 32$ \\
\hline
\end{tabular}


considered the drug of choice for Campylobacter treatment [1], may be clinically less effective for Campylobacter-like organism infections such as $H$. fennelliae and $H$. cinaedi. Little is known about the antimicrobial resistance mechanisms of $H$. fennelliae. Mutations of the gyrase and 23S rRNA genes may be responsible for decreased susceptibility to quinolones and macrolides, respectively [10]. However, decreased susceptibility to low MIC macrolide levels were mentioned in a previous study [13]. The $H$. fennelliae isolate from our study exhibited a high MIC to macrolides, warranting further molecular characterization to explore other resistance mechanisms.

The genotyping confirmation of this $H$. fennelliae isolate was performed by sequencing the cpn60 gene, and the result was submitted to NCBI under the accession number MG696736. A phylogenetic tree analysis (Fig. 1) divided Helicobacter and Campylobacter strains into seven distinct groups (cut-off of 90\%). The MG696736 entry was classified as group IV, which was $97.2 \%$ similar to H. fennelliae ATCC 35684, whereas Campylobacter species was classified as group VII, which is distinct from the Helicobacter group VI (cut-off of 90\%).

H. fennelliae was suggested as a significant pathogen associated with human gastroenteritis; however, its prevalence and antimicrobial resistant profile might be considerably underestimated due to inadequate isolation and identification methods [14]. To the best of our knowledge, this is the first report of a macrolide and quinolone resistant $H$. fennelliae identified in a young Cambodian child asymptomatic for intestinal infection. This isolate resembles $H$. fennelliae, which was previously identified in a boy suffering gastroenteritis and is also isolated from dog specimens [15]. With the introduction of the 'Cape Town Protocol', H. fennelliae may be isolated from stool and blood culture in an $\mathrm{H}_{2}$-rich microaerophilic atmosphere. Prior evidence indicated that Helicobacter species related to H. fennelliae were isolated from blood of a young child suffering diarrhea symptoms [16]. Nevertheless, the nucleotide sequences of H. fennelliae obtained from blood and stool were not significantly different [17]. Unfortunately blood samples were not available from the child in this study, so that comparison was not achievable. H. fennelliae was predominantly isolated from children who presented with diarrheal symptoms, although stools from asymptomatic diarrheal children with asthma and/or failure to thrive (FTT) were also positive for H. fennelliae [16, 17]. Another possible explanation of this $H$. fennelliae finding in stool of asymptomatic diarrheal Cambodia

\section{$\%$ Similarity}

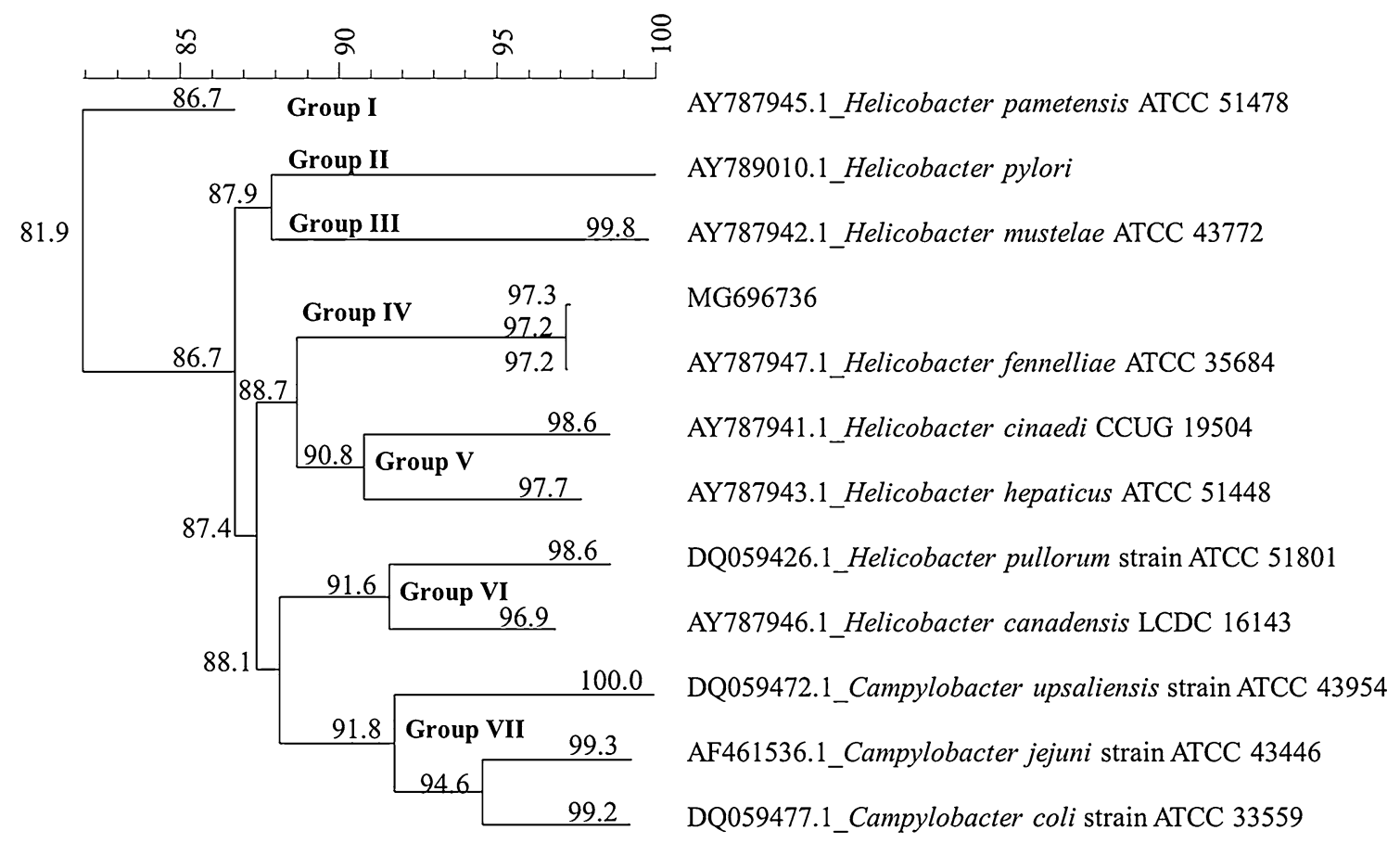

Fig. 1 Neighbor-joining phylogenetic trees based on partial cpn60 gene sequences (555 bp) of Helicobacter strain (MG696736), as compared to cpn60 sequences of other Helicobacter, and Campylobacter strains in cpnDB database 
child could relate to breastfeeding. Evidence suggests that maternal milk contains a variety of functionally bioactive agents from her innate immune system [18], as well as a mechanism to influence microbial changes in the infant's gastrointestinal system [19]. As a result of widespread breastfeeding campaigns in the developing world, this may play an important role in the level of asymptomatic carriage within a community $[18,20]$. The association between asymptomatic carriage and diarrheal pathogens such as Salmonella, E. coli O157 and Campylobacter was previously reported in outbreaks elsewhere [20]. Identification of an antibiotic resistant $H$. fennelliae strain from an asymptomatic diarrheal person would probably be transmitted into local communities and environmental contamination. Hence, the public health significance of resistant pathogens in human feces warrants effective monitoring to prevent disease outbreaks.

In conclusion, phenotypic and genotypic assessments confirmed that $H$. fennelliae was isolated from a nondiarrheal stool sample of a Cambodian child suffering from fever with cough and convulsion. The supplement media, incubation atmosphere, and incubation period utilized permitted culture, isolation, and identification of $H$. fennelliae. The high MICs values against macrolides (AZM, ERY) and quinolones (NAL, CIP) indicated these are less effective against $H$. fennelliae. This isolate should be further characterized to better understand associated resistance mechanisms.

\section{Authors' contributions}

WL and SR participated in the conception and design of the study. SR, PW, and CS performed the laboratory work. NS was clinical coordinator and subject enrollment. WL and OS analyzed the data and wrote the manuscript. SL and LC coordinated and fully supported this study in Cambodia. LB and JC contributed to the analysis and helped in writing the manuscript. All authors read and approved the final manuscript.

\section{Author details \\ ${ }^{1}$ Department of Enteric Diseases, Armed Forces Research Institute of Medical Sciences, 315/6 Rajvithi Road, Bangkok 10400, Thailand. ${ }^{2}$ Battambang Referral Hospital, PrekMohatep Village, SvayPor Commune, Battambang, Cambodia. \\ ${ }^{3}$ Armed Forces Research Institute of Medical Sciences, 18.118 Street Sangkat Mettapheap Khan 7 Makara, Phnom Penh, Cambodia. ${ }^{4}$ Communicable Dis- ease Control Department, Ministry of Health, 151-153, Kampuchea KromBlvd, Phnom Penh, Cambodia.}

\section{Acknowledgements}

We thank David Saunders, Brett E. Swierczewski, Carl J. Mason, SokVannara, Koy Lenin and Prom Satharath for supervision of this surveillance study. We thank AFRIMS Enteric Diseases Department Staff, Bangkok, Thailand and Battambang Referral Hospital \& AFRIMS-CNM Staff, Battambang, Cambodia, for their assistance and kind support.

\section{Competing interests}

The authors declare that they have no competing interests.

\section{Authors' disclaimers}

Material has been reviewed by the Walter Reed Army Institute of Research. There is no objection to its presentation and/or publication. The opinions or assertions contained herein are the private views of the author, and are not to be construed as official, or as reflecting true views of the Department of the Army or the Department of Defense.

\section{Availability of data and materials}

Data sharing not applicable to this article.

\section{Consent for publication}

Not applicable.

Ethics approval and consent to participate

The study protocol was in accordance with ethical guideline of the 'Code of Federal Regulations, Title 32, Part 219: Protection of Human Subjects' and was approved by the Review Board at National Ethics Committee for Health Research, Phnom Penh, Cambodia and Walter Reed Army Institute of Research (WRAIR), Silver Spring, MD, USA.

\section{Funding}

The study is supported by the Armed Forces Health Surveillance Branch (AFHSB) and it's GEIS (Global Emerging Infectious Disease Surveillance and Response) Section.

\section{Publisher's Note}

Springer Nature remains neutral with regard to jurisdictional claims in published maps and institutional affiliations.

Received: 11 April 2018 Accepted: 19 May 2018

Published online: 30 May 2018

\section{References}

1. Totten PA, Fennell CL, Tenover FC, Wezenberg JM, Perine PL, Satamm WE, et al. Campylobacter cinaedi (sp.nov.) and Campylobacter fennelliae (sp. Nov): two new Campylobacter species associated with enteric diseases in homosexual men. J Infect Dis. 1985;151(1):131-9.

2. James HJ, Michael AP, Karen CC, Guido FMLL, Sandry SR, David WW. Manual of clinical microbiology. Washington DC: American Society for Microbiology press; 2015.

3. O'Rourke JL, Grehan M, Lee A. Non-pylori Helicobacter species in humans. Gut. 2001;49(5):601-6.

4. Hill JE, Paccagnellla A, Law K, Melito PL, Woodward DL, Price L, et al. Identification of Campylobacter spp. and discrimination from Helicobacter and Arcobacter spp. by direct sequencing of PCR-amplified cpn60 sequences and comparison to $\mathrm{cpnDB}$, a chaperonin reference sequence database. J Med Microbiol. 2006;55(4):393-9.

5. Meng CY, Smith BL, Bodhidatta L, Richard SA, Vansith K, Thy B, et al. Etiology of diarrhea in young children and patterns of antibiotic resistance in Cambodia. Pediatr Infect Dis J. 2011;30(4):331-5.

6. Garcia LS. Clinical microbiology procedures handbook. 3rd ed, Washington, D.C.: ASM Press; 2010. P. 3.8.1.1-3.8.2.16 and 3.17.1.1-3.18.2.1.

7. Bullman S, O'Leary J, Corcoran D, Sleator RD, Lucey B. Molecular-based detection of non-culturable and emerging campylobacteria in patients presenting with gastroenteritis. Epidemiol Infect. 2012;140(4):684-8.

8. Chaban B, Musil KM, Himsworht CG, Hill JE. Development of cpn60-based Real-time quantitative PCR assays for the detection of 14 Campylobacter species and application to screening of canine fecal samples. Appl Environ Microbiol. 2009;75(10):3055-61.

9. Roop RM II, Smibert RM, Johnson JL, Krieg NR. Campylobacter mucosalis (Lawson, Leaver, Pettigrew, and Rowland 1981) comb. nov.: emended description. Int J Syst Bacteriol. 1985;35(1):189-92.

10. Rimbara E, Mori S, Kim H, Matsui M, Suzuki S, Takahashi S, et al. Helicobacter cinaedi and Helicobacter fennelliae transmission in a hospital from 2008 to 2012. J Clin Microbiol. 2013;51(7):2439.

11. Fujiya Y, Nagamatsu M, Tomida J, Kawamura Y, Yamamoto K, Mawatari M, et al. Successful treatment of recurrent Helicobacter fennelliae bacteraemia by selective digestive decontamination with kanamycin in a lung cancer patient receiving chemotherapy. JMM Case Rep. 2016;3(5):e005069. 
12. Kawamura Y, Tomida J, Morita Y, Fujii S, Okamoto T, Akaike T. Clinical and bacteriological characteristics of Helicobacter cinaedi infection. J Infect Chemother. 2014;20(9):517-26.

13. Hsueh PR, Teng LJ, Hung CC, Chen YC, Yang PC, Ho SW, et al. Septic shock due to Helicobacter fennelliae in a non-human immunodeficiency virusinfected heterosexual patient. J Clin Microbiol. 1999;37(6):2084-6.

14. Lastovica AJ. Emerging Campylobacter spp.: the tip of the iceberg. Clin Microbiol Newsl. 2006;28(7):49-56.

15. Burnens AP, Stanley J, Schaad UB, Nicolet J. Novel Campylobacter-like organism resembling Helicobacter fennelliae isolated from a boy with gastroenteritis and from dogs. J Clin Microbiol. 1993;31(7):1916-7.

16. Tee W, Hinds S, Montgomery J, Dyall-Smith ML. A probable new Helicobacter species isolated from a patient with bacteremia. J Clin Microbiol. 2000;38(10):3846-8.

17. Smuts HE, Lastovica AJ. Molecular characterization of the $16 \mathrm{~S}$ rRNA Gene of Helicobacter fennelliae isolated from stools and blood cultures from paediatric patients in South Africa. J Pathog. 2011. https://doi. org/10.4061/2011/217376.

18. Morrow AL, Ruiz-Palacios GM, Altaye M, Jiang X, Guerrero ML, MeinzenDerr JK, et al. Human milk oligosaccharides are associated with protection against diarrhea in breast-fed infants. J Pediatr. 2004;145(3):297-303.

19. Ogbo FA, Agho K, Ogeleka P, Woolfenden S, Page A, Eastwood J, Global Child Health Research Interest Group. Infant feeding practices and diarrhoea in sub-Saharan African countries with high diarrhoea mortality. PLoS ONE. 2017;12(2):e0171792. https://doi.org/10.1371/journ al.pone.0171792.

20. Quilliam RS, Cross P, Williams AP, Edwards-Jones G, Salmon RL, Rigby D, et al. Subclinical infection and asymptomatic carriage of gastrointestinal zoonoses: occupational exposure, environmental pathways, and the anonymous spread of disease. Epidemiol Infect. 2013;141(10):2011-21.
Ready to submit your research? Choose BMC and benefit from:

- fast, convenient online submission

- thorough peer review by experienced researchers in your field

- rapid publication on acceptance

- support for research data, including large and complex data types

- gold Open Access which fosters wider collaboration and increased citations

- maximum visibility for your research: over $100 \mathrm{M}$ website views per year

At BMC, research is always in progress.

Learn more biomedcentral.com/submissions 